\title{
28 Research Square \\ Development of an Optimal Relief Method for the Palatal Plate by Stress Analysis
}

\section{Tomoko Mukai ( $\nabla$ t.mukai@dent.showa-u.ac.jp )}

Showa University

\section{Yuji Sato}

Showa University

Osamu Shimodaira

Showa University

Junichi Furuya

Showa University

Akio Isobe

Showa University

Tomoka Omori

Showa University

\section{Research Article}

Keywords: Palatal mucosa, Relief, Simulation, Three-dimensional finite element analysis, Stress distribution, Borderline

Posted Date: September 28th, 2021

DOl: https://doi.org/10.21203/rs.3.rs-923623/v1

License: (c) (i) This work is licensed under a Creative Commons Attribution 4.0 International License.

Read Full License

Version of Record: A version of this preprint was published at BMC Oral Health on December 1st, 2021. See the published version at https://doi.org/10.1186/s12903-021-02014-z. 


\section{Abstract}

Background: A maxillary-palate-shaped device for simultaneous measurement of bite force and palatal mucosal subsidence at the time of pain onset in dentate persons has been developed. However, palatal mucosal stress analysis in a simulation based on three-dimensional finite element analysis is effective for objective and efficient evaluation of various types of denture-supporting mucosa. Recently plate dentures are not easily modified after the completion of these dentures, so it is essential to effectively assess the sites and magnitude of relief at the time of preparation. However, there is considerable variation in the magnitude of optimal relief and relief range, and there are no guidelines that present these clearly, leading the surgeon to decide subjectively. Thus, this study aimed to develop an optimal relief method to improve the stress bearing capacity of the palatal mucosa.

Objectives: The objective of this study, the borderline was set in steps. The changes in stress distribution in the palatal mucosa due to the selective relief of stresses above the borderline were evaluated using a three-dimensional finite element simulation. The purpose of this study was to develop an optimal relief method to improve the bearing capacity of the palatal mucosa.

Methods: The objective of this study, the borderline, was set in steps. A three-dimensional finite element model for the pseudopalatal plate was prepared and used to evaluate the changes in stress distribution in the palatal mucosa due to the selective relief of stresses above the borderline. The resulting data were used to develop the optimal relief method.

Results: In the relief model with a borderline of $0.04 \mathrm{MPa}$ or higher, the distribution volume at which high stress of $0.20 \mathrm{MPa}$ or higher is generated was approximately about $800 \% \bigotimes$ of that with the no-relief model, and in the relief model with a borderline of $0.06 \mathrm{MPa}$ or higher, the respective ratio was

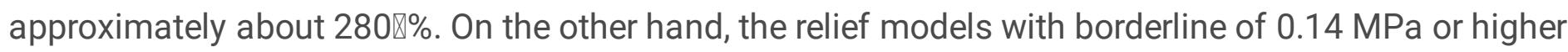
were about $60 \%$. In the mid-palatal relief model, the distribution volume at which stress of $0.20 \mathrm{MPa}$ or higher was generated was $180 \%$ of that in the relief model.

Conclusions: The supportive strength of plates can be increased by selectively applying optimal relief rather than standard relief, allowing for easier and more effective plate-denture treatment.

\section{Background}

With the aging population in Japan, there is an increasing demand for removable denture prosthetics. ${ }^{1}$ High-quality plate-denture treatment is linked to an increased quality of life for patients. For plate-denture treatment, objective evaluation of the properties of the denture-supporting mucosa is important.

Recently, plate dentures with frameworks of cobalt-chromium alloy and zirconia have been widely used for their increased durability and comfort while in use. ${ }^{2,3}$ However, after the completion of these dentures, it is difficult to modify them by cutting, and it is therefore essential to assess the sites and magnitude of relief sufficiently at the time of preparation. In practice, in connection with complete denture treatment in 
the past, as a part of denture design, relief has been provided in the mid-palatal area. This relief is an important means of preventing pain due to the denture plate in the region of mucosal thinning, ensuring denture stability, and preventing denture damage, but also preventing compression injury to the nerves and blood vessels. ${ }^{4}{ }^{5}$ However, there is considerable variation in the magnitude of optimal relief and relief range, and there are no guidelines that present these clearly, leading the surgeon to decide subjectively.

To optimize denture design for individual patients, a system has been developed by fitting a strain gauge to an ultrasonic thickness gauge so that changes in load and mucosal thickness causing pain are measured simultaneously. ${ }^{6}$ The relationships between the properties of the denture-supporting mucosa (thickness and elasticity) and the pain threshold (pressure, degree of subsidence, and compression rate) in dentate and edentulous persons were analyzed. ${ }^{7,8}$ In addition, to apply this system clinically, a maxillary-palate-shaped device for simultaneous measurement of bite force and palatal mucosal subsidence at the time of pain onset in dentate persons has been developed. Using this device, the relationships between bite force and palatal mucosal subsidence in dentate persons were analyzed simultaneously, and the effects of palatal relief on palatal-mucosal-supporting strength were clarified. ${ }^{9}$ However, palatal mucosal stress analysis in a simulation based on three-dimensional finite element analysis is effective for objective evaluation of various types of denture-supporting mucosa. ${ }^{10}$

In this study, the borderline was set in steps. The changes in stress distribution in the palatal mucosa due to the selective relief of stresses above the borderline were evaluated using a three-dimensional finite element simulation. The purpose of this study was to develop an optimal relief method to improve the stress bearing capacity of the palatal mucosa.

\section{Methods}

\section{Subject and simulation range}

The subject was a dentate person with no abnormality in the palatal mucosa or presence of a marked tori. This person participated in previous studies in which mucosal elasticity and thickness were measured. Further, a three-dimensional finite element model was prepared. Similar to previous studies, the simulation range was defined as the palatal mucosa extending on the maxilla from a position mesial to the first molars on the left and right side to a position distal to the second molars on the left and right. ${ }^{9 \text {, }}$ ${ }^{10}$ The study was performed in accordance with the principles of the Declaration of Helsinki. The informed consent form was approved by the Ethics Committee of Showa University (approval number 2014-036).

\section{Establishment of three-dimensional finite element models}

\section{1) Establishment of the pseudopalatal plate model}

The pseudopalatal plate of the device for simultaneous measurement of the palatal region was prepared using X-radiographically observable scanning resin, and computed tomography was performed. A three- 
dimensional finite element model for the pseudopalatal plate was created from the computed

tomography data obtained using three-dimensional finite element analysis software (Mechanical Finder ${ }^{\circledR}$; Research Center of Computational Mechanics, Tokyo, Japan; Fig. 1). This was referred to as the no-relief model. $^{10}$

\section{2) Relief settings}

\section{(1) Setting the borderline for the stress}

In a previous study, the pain generating bite force was measured as $111 \mathrm{~N} .{ }^{10}$ This force was applied to the center of the no-relief model, and the value of the stress thus generated in the palatal mucosal model at that time, $0.02 \mathrm{MPa}$, was set as one end of the range for bite force in the simulation, with $0.20 \mathrm{MPa}$ as the other end. In other words, the bite forces set were $0.04,0.06,0.08,0.10$, and $0.14 \mathrm{MPa}$. The stress generation ranges at each bite force or higher were considered to be the target ranges for relief (Fig. 2).

\section{(2) Relief in ranges within which stress is generated at borderline or higher}

Based on the range in the no-relief model where stresses above the borderline were generated, this model was trimmed. Then, a relief of $0.25 \mathrm{~mm}^{11}$ was applied to this trimmed area to create the outline of the pseudo palatal floor model with relief. Each of the resulting models were categorized as the $0.04 \mathrm{MPa}$ or higher, $0.06 \mathrm{MPa}$ or higher, $0.08 \mathrm{MPa}$ or higher, $0.10 \mathrm{MPa}$ or higher, and 0.14 MPa or higher relief models (Fig. 2).

\section{(3) Single relief for the general mid-palatal area}

On the basis of the no-relief model, relief was applied to breadths of 10 and $0.25 \mathrm{~mm}$ in the maxillary mid-palatal area, giving the external form of the pseudopalatal plate model a single relief applied to the maxillary mid-palatal area. This is referred to as the mid-palatal relief model (Fig. 2).

\section{Setting the external form of the palatal mucosa model}

The mucosal surface in the pseudopalatal plate model was divided into 14 segments consistent with the measurement sites, and the measured palatal mucosal thickness was added as an element. In addition, the segments were edited to ensure smoothness. ${ }^{10}$

\section{Mesh formation}

Taking the elements composing the mesh to form a tetrahedron, 88,951 nodal points were noted and divided with a total number of 475,962 elements. In addition, both the pseudopalatal plate model and the palatal mucosa model were considered to be homogeneous and isotropic linear elastic structures.

\section{Analysis conditions}

1) Setting physical values 
The physical properties of the resin in a humid environment were evaluated using a pseudopalatal plate model. The elasticity was assumed to be $2,650 \mathrm{MPa},{ }^{12}$ and the Poisson's ratio was $0.3 .{ }^{13}$ The elasticity calculated from the measurements of the subject was applied to the palatal mucosa model. ${ }^{10}$

\section{2) Loading conditions}

The loading was vertical onto the center of the pseudopalatal plate. With respect to the load, when the subject developed pain, the bite force was $111 \mathrm{~N} .^{10}$

\section{3) Restraint conditions}

The uppermost surface of the palatal mucosa model was assumed to adhere to the maxillary bone, and the model was fully constrained. ${ }^{10}$

\section{4) Boundary conditions}

The boundary conditions in the pseudopalatal plate model and palatal mucosa model were considered as the adhesion conditions. ${ }^{10}$

\section{Analysis target}

In the palatal mucosa model, the volume of the range within which von Mises stress was generated at each borderline or higher was calculated, and the results were compared between each of the conditions, with and without relief.

\section{Results}

\section{Comparison of von Mises stress generated in relief and no-relief palatal mucosa models}

The distributions of von Mises stress generated in the relief and no-relief palatal mucosa models are shown in Fig. 3.

In the relief model with borderline at $0.04 \mathrm{MPa}$ or higher and $0.06 \mathrm{MPa}$ or higher, the stress distribution was higher toward the front of the mid-palatal area than in the no-relief model. In the relief model with borderline at 0.08 or higher, 0.10 or higher, and $0.14 \mathrm{MPa}$ or higher, no marked changes were found in comparison with the no-relief model. In addition, in the mid-palatal relief model, stress distribution was at the margin of the relief range and at both margins of the palatal plate.

\section{Distribution volume ratios for each stress values}

The distribution volume ratios for each stress value on the palatal mucosa are shown in Fig. 4 . With a borderline of $0.04 \mathrm{MPa}$ or higher and $0.06 \mathrm{MPa}$ or higher, the relief model was found to increase the distribution volume of stress at $0.20 \mathrm{MPa}$ or higher. In the relief model with a borderline of $0.08 \mathrm{MPa}$ or 
higher and $0.10 \mathrm{MPa}$ or higher, the stress distribution was at $0.14 \mathrm{MPa}$ or below. In the relief model with a borderline of $0.14 \mathrm{MPa}$ or higher, the stress distribution at $0.08 \mathrm{MPa}$ or higher decreased, and stress at $0.06 \mathrm{MPa}$ or lower increased. In the mid-palatal relief model, the distribution volume of stress at $0.04 \mathrm{MPa}$ or higher increased in comparison with that in the no-relief model. In addition, a comparison of the relief models with each borderline showed maximum increases in stress distribution volume between 0.06 and $0.12 \mathrm{MPa}$.

\section{Distribution volume ratios for each stress value in the relief model}

The distribution volumes in the relief model relative to the situation when the distribution volume for each stress value in the no-relief model was considered to be $100 \%$ are shown in Fig. 5 . In the relief model with a borderline of $0.04 \mathrm{MPa}$ or higher, the distribution volume at which high stress of $0.20 \mathrm{MPa}$ or higher was generated was approximately $800 \%$ of that with the no-relief model, and in the relief model with a borderline of $0.06 \mathrm{MPa}$ or higher, the respective ratio was approximately $280 \%$. On the other hand, the relief models with borderline of $0.08 \mathrm{MPa}$ or higher, $0.10 \mathrm{MPa}$ or higher, and $0.14 \mathrm{MPa}$ or higher were less than $100 \%$, and among them, the relief models with values of $0.14 \mathrm{MPa}$ or higher were approximately $60 \%$. In the mid-palatal relief model, the distribution volume at which stress of $0.20 \mathrm{MPa}$ or higher was generated was $180 \%$ of that in the relief model.

\section{Discussion}

\section{Subject and simulation range}

The subject in this study was dentate, with healthy teeth and was selected as a reference to obtain useful data regarding the palatal mucosa of dentate patients before evaluating the denture-supporting mucosa of edentulous patients. As the present study covered only the mean palatal configuration of a single subject, it was confined to various aspects of the intraoral status of that subject, including the presence or absence of palatal tori, and palatal shape, with the primary focus being the development of analysis methods. In the future, it will also be necessary to investigate the differences due to palatal shape. ${ }^{9}$

In addition, the simulated relief model is currently used in practice for creating pseudopalatal plates that reflect a relief based on each borderline in CAD/CAM that was output from the STL data. These plates were combined with a simultaneous measurement device ${ }^{9}$ and were used to verify the simulated validity with subjects.

\section{Setting the borderline}

In this study, the borderline for the simulation was set between 0.02 , which was the level of stress generated in the palatal mucosa model, and $0.20 \mathrm{MPa}$, and the stress distribution was analyzed. It is difficult to set stress values for the onset of pain, but pain is thought to occur readily at relatively high stress values. 


\section{Finite element analysis methods and analysis models}

Analysis based on finite elements enables the partitioning of elements in the model, detailed setting of loading conditions and physical properties, and accurate simulation based on the introduction of temporal elements to the linear analysis and dynamic analysis. However, if analysis is performed with more detailed conditions set, considerable time is required; therefore, for commercial use, in order to have a general appreciation of the entire tendency at the initial stages of structural design, sometimes only a first-order analysis is performed, that is, an approximate analysis, with the details simplified. ${ }^{14}$ In the present study, the stress distribution was analyzed using the finite element analysis (FEA) model, and a simulation was performed for the stress distribution on the denture-supporting mucosa when relief was applied to ranges of the borderline and higher.

\section{Effects of relief on the palatal mucosa}

In this study, with a relief model at $0.04 \mathrm{MPa}$ or higher and a mid-palatal relief model, there were increases in the high-stress distribution volume in comparison with that in a no-relief model (Figs. 4, 5). With excessive relief and standard relief, respectively, the supportive surface areas of plates outside the relief range was decreased and excessive local stress was generated; hence, pain is considered to occur readily. On the other hand, in relief models at $0.14 \mathrm{MPa}$ or higher, stress was dispersed homogeneously throughout the palatal mucosa. If relief can be applied locally, restricted to the sites of high stress generation, it is probable that by making the stress consistent, the supportive strength of the plate can be increased.

In summary, the findings of this study suggest that relief applied based on the dentist's subjective judgment and experience is not necessarily effective.

The plans for future research include applying structurally optimized techniques, making stress values variable, and repeatedly verifying the effectiveness of relief.

\section{Conclusions}

In this study, the threshold values for the stress generated in the palatal mucosa were set, and by selectively applying relief for the stress ranges generated at or above each of these values, evaluation could be performed using a simulation involving three-dimensional finite element analysis of stress distribution on the palatal mucosa. This suggests that the supportive strength of plates can be increased by selectively applying optimal relief rather than standard relief.

\section{Abbreviations}

FEA: finite element analysis

\section{Declarations}


Ethics approval and consent to participate: The study was performed in accordance with the principles of the Declaration of Helsinki. The informed consent form was approved by the Ethics Committee of Showa University (approval number 2014-036).

Consent for publication: Not applicable

Availability of data and materials: Please contact author for data request.

Competing interests: The authors declare that they have no competing interests.

\section{Funding:}

This work was supported by JSPS KAKENHI Grant Number 16K20513.

\section{Authors' contributions:}

TM contributed to the study design/conception, material preparation, and execution of the threedimensional finite element models. YS provided supervision, corrected the manuscript, and reviewed the progress of the study. OS contributed to the study design/conception and material preparation. JF and Al interpreted the results and revised the manuscript. TO contributed to the draft preparation and execution of the three-dimensional finite element models.

Acknowledgements: The authors would like to thank the staff of the Department of Geriatric Dentistry, Showa University.

\section{References}

1. Sato Y, et al. Estimation of denture patients' absolute number in elderly from survey of dental diseases and vital statistics. Jpn J Dent Practice Administration. 2014;49:162-167 [in Japanese].

2. Tanaka S. Digital dentistry Changes Prosthodontics. Journal of the Showa Medical Association 2015;75:38-45 [in Japanese].

3. Hiraoka H. Clinical practice of the zirconia denture base. The International Journal of Dental Technology 2015;43:408-421.

4. Academy of denture prosthetics: glossary of prosthodontic terms. J Prosthet Dent 2005;94:10-81.

5. Masatoshi I, Misao K, Sayumi I, et al. Pressure dynamics in the trays caused by differences of the various impression materials and thickness of the relief in the maxillary edentulous model. $J$ Prosthodont Res 2016;60:123-130.

6. Takeuchi S, Sato Y, Sato Y, et al. Measurements of viscoelasticity of oral mucosa -establishing a simultaneous measurement method for load and change of the mucosa thickness- Ann Jpn Prosthodont Soc 2010;2:70-77 [in Japanese].

7. Isobe A, Sato Y, Kitagawa N, Shimodaira O, Hara S, Takeuchi S. The influence of denture supporting tissue properties on pressure-pain threshold -measurement in dentate subjects. J Prosthodont Res 
2013;57:275-283.

8. Kotani Y. Relationship between palatal mucosa properties and pressure-pain threshold in young dentulous and elderly edentulous subjects. J J Gerodont 2015;30:68-79.

9. Tanaka S, Sato Y, Kitagawa N, et al. Effect of relief at the median palatal plate on denture-supporting ability. Int J Prosthodont Endod 2017;7:117-123.

10. Omori T, Sato Y, Kitagawa N, et al. Effects of palatal plate loading on mucosa development in threedimensional finite element model. Int J Prosthodont Endod 2020;10:50-56.

11. Ishinabe S. Mucosal thickness of the denture foundation under occlusal force. J Jpn Prosthodont Soc 1991;35:111-124 [in Japanese].

12. Sato $Y$, Abe $Y$, Okane H, Tsuga K. Finite element analysis of stress relaxation in soft denture liner. J Oral Rehabil 2000;27:660-663.

13. Kanbara R, Nakamura Y, Ochiai KT, Kawai T, Tanaka Y. Three-dimensional finite element stress analysis: the technique and methodology of non-linear property simulation and soft tissue loading behavior for different partial denture designs. Dent Mater J 2012;31:297-308.

14. Omori M, Sato Y, Kitagawa N, Shimura Y, Ito M. A biomechanical investigation of mandibular molar implants: reproducibility and validity of a finite element analysis model. Int J Implant Dent 2015;1:10.

\section{Figures}

(A)

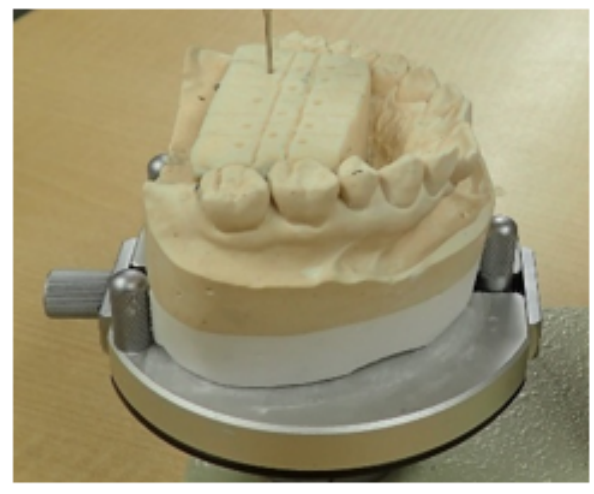

(C)

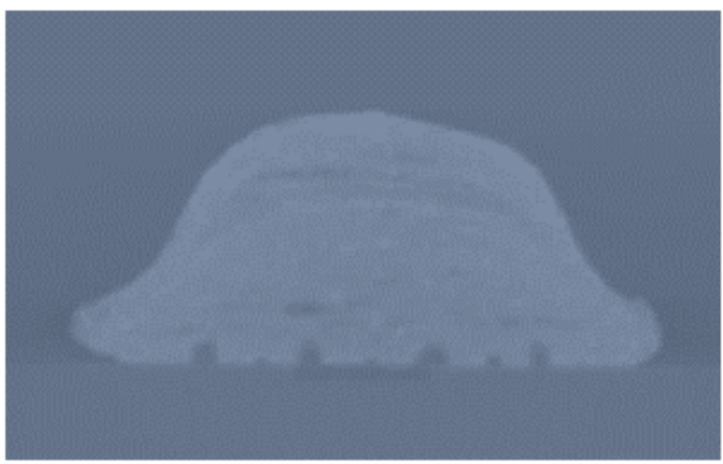

(B)

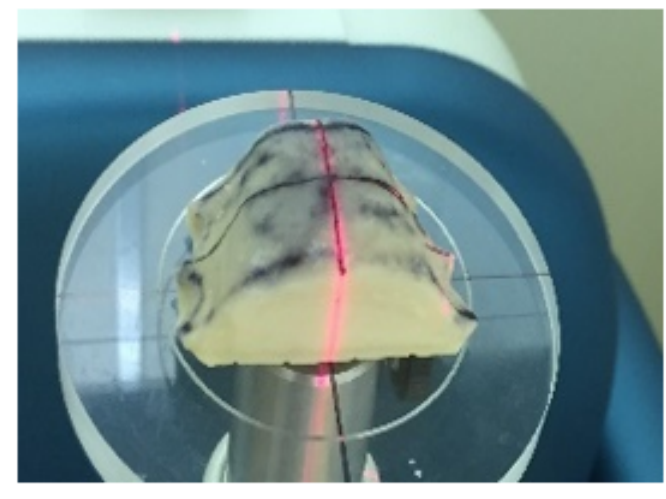

(D)

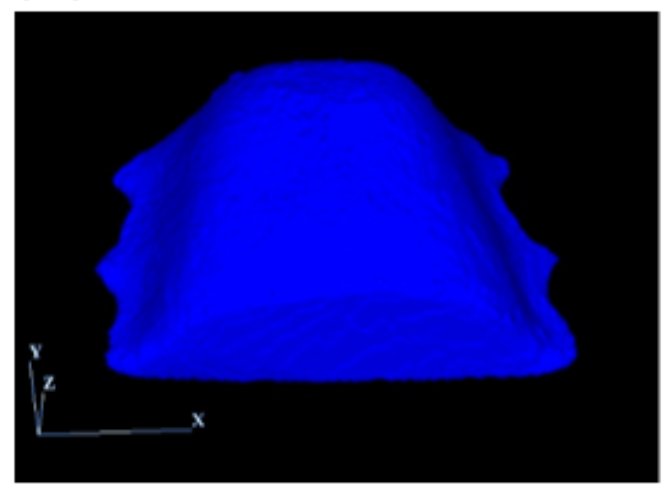


Figure 1

Constructing the three-dimensional finite element model (A) Pseudopalatal plate using scanning resin; (B) CT imaging; (C) captured by 3D-FEA software; (D) three-dimensional finite element model of the pseudopalatal plate
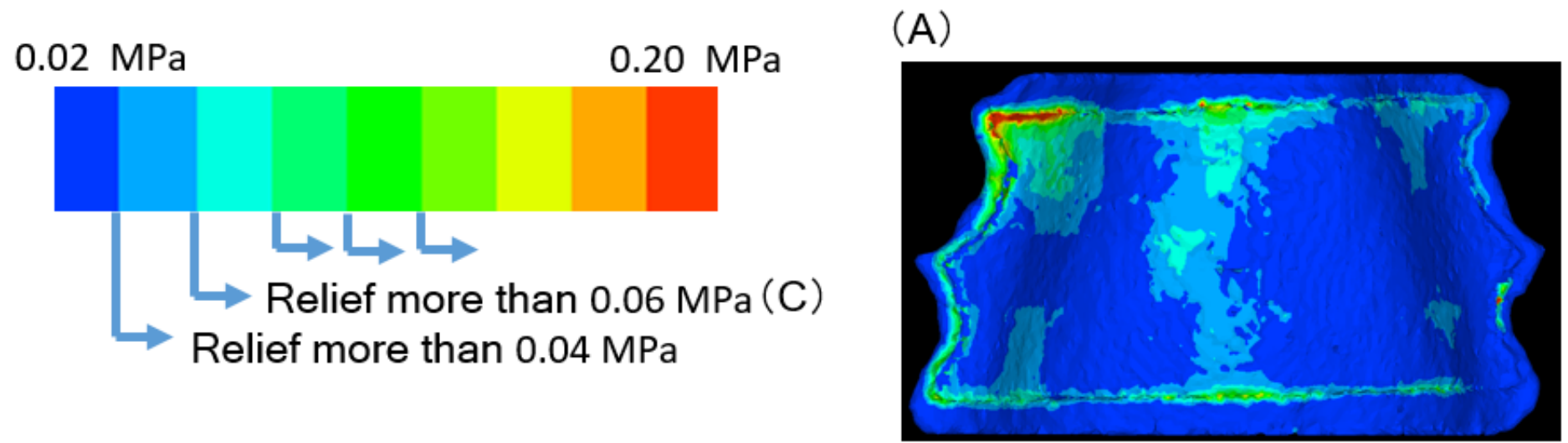

(B)

(C)
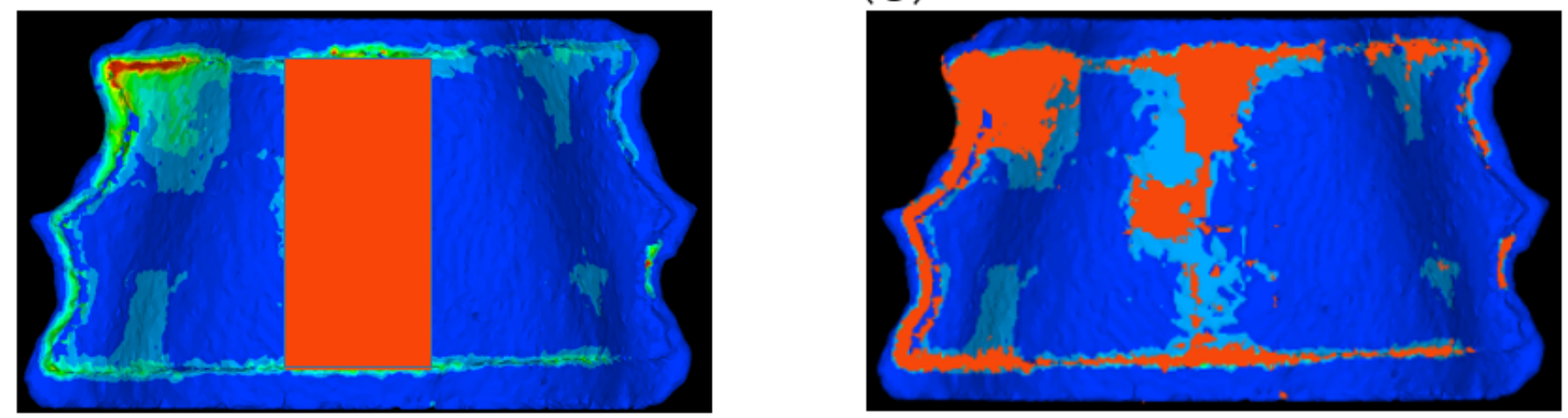

Figure 2

Relief setting (A) No relief; (B) mid-palate relief; (C) relief more than $0.06 \mathrm{MPa}$ 

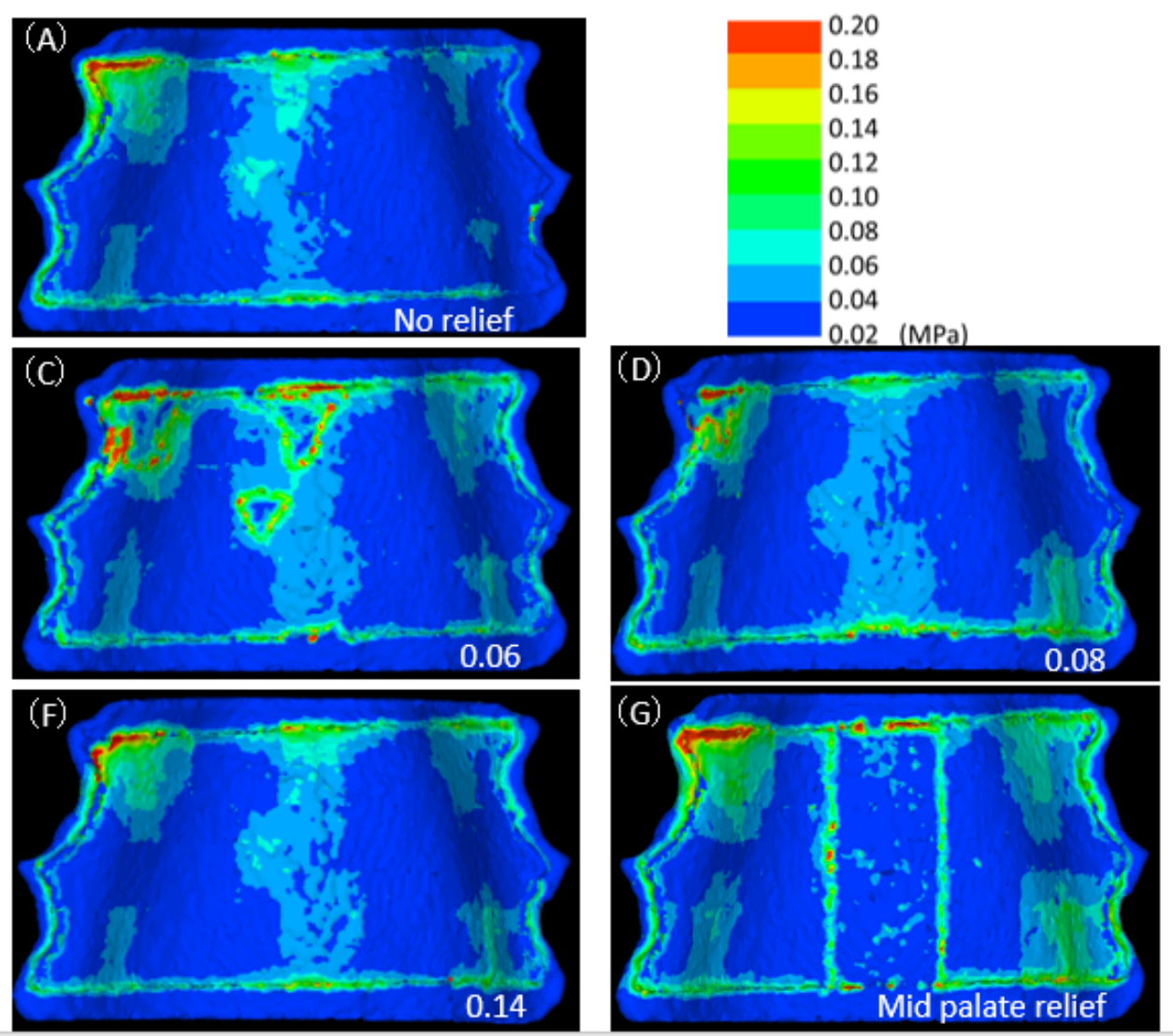

\section{Figure 3}

Distribution map of von Mises stress (A) No relief; (B) relief more than $0.04 \mathrm{MPa}$; (C) relief more than 0.06 $\mathrm{MPa}$; $(\mathrm{D})$ relief more than $0.08 \mathrm{MPa}$; $(\mathrm{E})$ relief more than $0.10 \mathrm{MPa}$; $\mathrm{F}$ ) relief more than $0.14 \mathrm{MPa}(\mathrm{G})$ midpalate relief. 


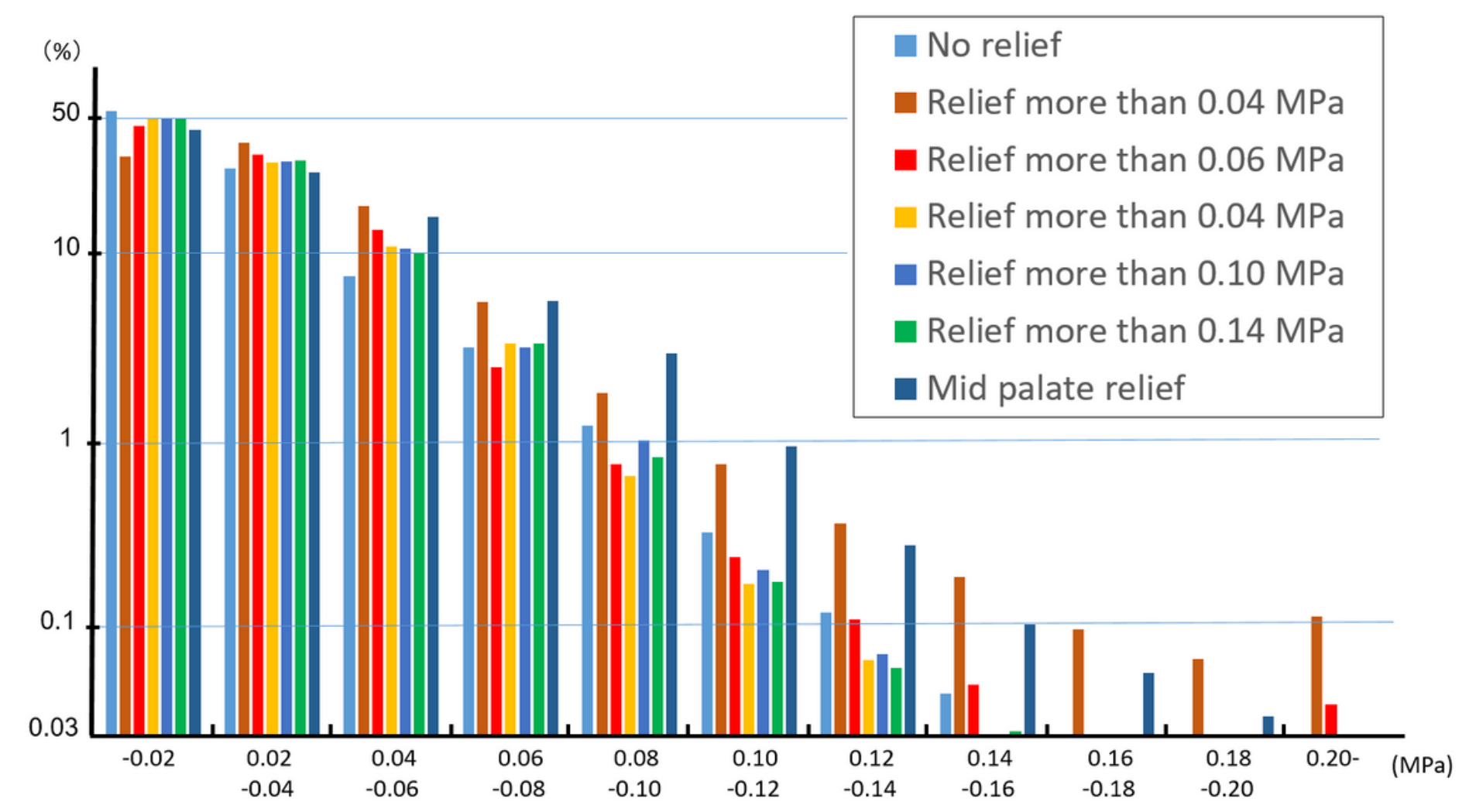

Figure 4

Volume ratio of stress distribution at each stress value

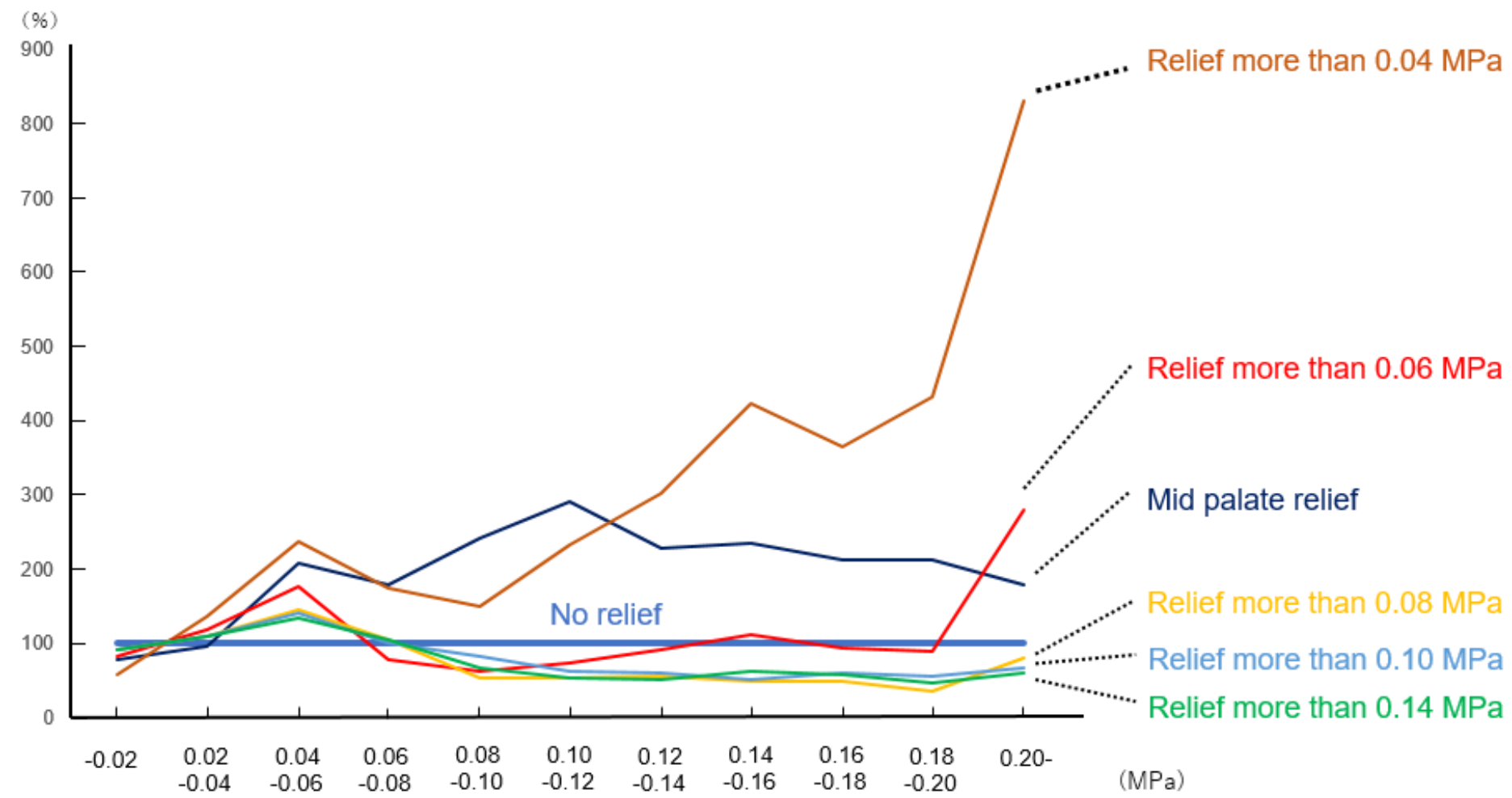

Figure 5 
Comparison of relief model and no-relief model

Page 13/13 